\title{
Efeito agudo do intervalo passivo e do alongamento no desempenho de séries múltiplas
}

\author{
Acute effect of passive rest intervals and stretching exercise \\ on multiple set performance
}

\author{
Antonio Cláudio do Rosário Souza ${ }^{1,2}$ \\ Carmen Lúcia Borges Bastos ${ }^{1,2}$ \\ Maria de Nazaré Dias Portal \\ Belmiro Freitas de Salles \\ Thiago Matassoli Gomes ${ }^{4,5}$ \\ Jefferson da Silva Novaes 4,5
}

1 Universidade Castelo Branco. Mestrado em Ciência da Motricidade Humana. Rio de Janeiro, RJ. Brasil.

2 Universidade Trás-osMontes e Alto Douro. Programa de Doutorado em Ciência do Desporto. Vila Real. Portugal.

3 Universidade do Estado do Rio de Janeiro. Laboratório de Pesquisa em Microcirculação. Rio de Janeiro, RJ. Brasil.

4 Universidade Federal do Rio de Janeiro. Programa de Pós-Graduação Stricto Sensu em Educação Física. Rio de Janeiro, RJ. Brasil.

5 Universidade Federal do Rio de Janeiro. Grupo de Pesquisa em Treinamento de Força. Escola de Educação Física e Desportos. Rio de Janeiro, RJ. Brasil.

Recebido em 28/09/08 Aprovado em 05/05/09
Resumo - Este estudo verificou o efeito agudo de intervalos passivos e do alongamento estático entre as séries sobre o número de repetições máximas (RM), percepção subjetiva de esforço (PSE) e volume total (VT) do número de repetições em um protocolo de séries múltiplas com sobrecarga ajustada pelo teste de 8RM. Participaram 14 homens $(24,4 \pm 2,1$ anos; 79,1 $\pm 7,1 \mathrm{~kg} ; 175,4 \pm 5,6 \mathrm{~cm}$ ). O grupo de voluntários foi dividido de forma aleatória e nas duas primeiras visitas, realizaram o teste e o reteste de $8 \mathrm{RM}$ no supino reto (SR) e agachamento (AG). Nas duas sessões seguintes, os indivíduos foram divididos aleatoriamente em duas situações experimentais: a) teste de 8RM com intervalo passivo (GIP); b) teste de 8RM com exercício de alongamento (GAL). Foram realizadas três séries no SR e no AG com intervalos de dois minutos de forma passiva ou incluindo 30 segundos de alongamento. A ANOVA mostrou diferenças significativas $(\mathrm{p}<0,05)$ na segunda $(\mathrm{GIP}=$ $6 \pm 0,8 \times \mathrm{GAL}=5,2 \pm 1,0$ repetições) e terceira (GIP $=4,1 \pm 0,8 \mathrm{X} \mathrm{GAL}=3,3 \pm 0,6$ repetições) séries para o exercício $\mathrm{SR}$ e apenas na terceira série (GIP $=4,9 \pm 0,8 \mathrm{X}$ GAL $=4,2 \pm$ 1,0 repetições) para o AG. Na PSE, o teste de Wilcoxon mostrou diferenças significativas $(\mathrm{p}<0,05)$ entre todas as séries no SR e AG. Para o VT de RM, o teste t pareado mostrou diferenças significativas ( $\mathrm{p}<0,05)$ no SR ( $\mathrm{GIP}=18,3 \pm 1,5 \mathrm{X} \mathrm{GAL}=16,8 \pm 1,6$ repetições). Conclui-se que exercício de alongamento estático entre as séries pode provocar diminuição no desempenho da força em 8RM.

Palavras-chave: Força muscular; Intervalo; Alongamento; Percepção subjetiva de esforço.

Abstract - The objective of this study was to determine the acute effect of passive rest intervals and static stretching between resistance exercise sets on the number of maximal repetitions (RM), rating of perceived exertion (RPE), and cumulative number of repetitions in multiple sets with a workload adjusted by the 8RM test. Fourteen trained male subjects ( $24.4 \pm 2.1$ years; $79.1 \pm$ $7.1 \mathrm{~kg} ; 175.4 \pm 5.6 \mathrm{~cm})$ were studied. On the first two visits, the subjects were submitted to the test and 8RM re-test using chest press (CP) and squat (SQ) exercises. On the two subsequent visits, all subjects were randomly assigned to two experimental situations: a) 8RM test with a passive rest interval (PI); b) 8RM test with static stretching (SS). The subjects performed three sets of CP and SQ, intercalated with 2 minutes of passive rest or 30 seconds of static stretching. ANOVA revealed a significant decrease $(p<0.05)$ in the second $(P I=6 \pm 0.8 \times S S=5.2 \pm$ 1.0 repetitions) and third (PI $=4.1 \pm 0.8 \times$ SS $=3.3 \pm 0.6$ repetitions) sets for $\mathrm{CP}$ and only in the third set $(P I=4.9 \pm 0.8 \times$ SS $=4.2 \pm 1.0$ repetitions) for SQ. For RPE, the Wilcoxon test showed significant differences $(p<0.05)$ between all sets for CP and SQ. For the cumulative number of repetitions, the paired t-test revealed a significant decrease $(p<0.05)$ for $C P(P I=$ $18.3 \pm 1.5 \times$ SS $=16.8 \pm 1.6$ repetitions). These results indicate that static stretching between resistance exercise sets decreases $8 R M$ test performance.

Key words: Muscle strength; Rest interval; Stretching; Rating of perceived exertion. 


\section{INTRODUÇÃO}

O intervalo entre as séries é uma variável de suma importância na elaboração de um programa de treinamento de força (TF). Diferentes tipos de atividades durante o intervalo e diferentes tempos de recuperação entre as séries podem resultar em distintas respostas fisiológicas. Confirmando esta hipótese, alguns experimentos observaram que o intervalo de recuperação entre as séries pode ter influência sobre as adaptações dos sistemas neuromuscular e endócrino ${ }^{1,2}$. Em relação ao desenvolvimento de força nas séries subsequentes, tem-se demonstrado que intervalos passivos de três minutos ou menos (até 30 segundos) podem implicar reduções do número de repetições máximas (RM) durante a progressão das séries ${ }^{3,4}$.

Alguns estudos ${ }^{5-7}$, apesar de utilizarem metodologias bastante distintas com diferentes números de exercícios e variados protocolos de manutenção na posição de alongamento, demonstraram que ocorre uma diminuição no desempenho da força e da potência muscular quando estas são precedidas por exercícios de alongamento. Entretanto, outros estudos $^{8-11}$ demonstraram não haver influência significativa da flexibilidade realizada de forma estática no desempenho dos saltos verticais, nos testes isocinéticos e nos testes de contração voluntária máxima.

A fadiga durante o TF parece ter íntima relação com o tempo e tipo de intervalo adotado entre séries $^{12}$, tornando-se um elemento importante a ser considerado pelo profissional que o prescreve tanto na busca de melhores efeitos, quanto no aumento do potencial de adesão aos programas. Uma forma de controle da intensidade de esforço no TF e, consequentemente, de manipular as variáveis da prescrição de acordo com os níveis de fadiga desejáveis pode ser a percepção subjetiva de esforço (PSE). Porém, a utilização da PSE no TF é incomum, como demonstram poucas referências na literatura. Alguns estudos apresentam uma escala específica para o TF em adultos e crianças denominada OMNI-RES ${ }^{13,14}$ e apontam que a PSE pode refletir a intensidade de esforço na musculatura ativa durante o exercício. Porém, a literatura é escassa quanto à utilização da PSE para verificar a influência das diferentes variáveis metodológicas sobre a fadiga muscular localizada, tendo os estudos encontrados utilizado a escala de Borg (CR 10) adaptada para o $\mathrm{TF}^{12,15}$.

Após revisão da literatura pertinente ao assunto, foi encontrado apenas um estudo ${ }^{16}$ que procurou investigar o efeito de diferentes tempos e tipos de intervalos entre séries sobre o desempenho da força muscular. No entanto, nenhum experimento comparando a influência de intervalos passivos à realização de alongamentos específicos entre as séries sobre desempenho de força e da PSE em séries múltiplas pôde ser encontrado. Sendo assim, o objetivo deste experimento foi verificar o efeito agudo do intervalo passivo e do alongamento estático sendo realizados entre as séries, sobre o número de RM, sobre a resposta da PSE e sobre o volume total do número de repetições em um protocolo de séries múltiplas com sobrecarga ajustada pelo teste de 8RM realizado por homens treinados.

\section{PROCEDIMENTOS METODOLÓGICOS}

\section{Grupo de voluntários}

O estudo foi composto por 14 homens voluntários $(24,4 \pm 2,1$ anos; 79,1 $\pm 7,1 \mathrm{~kg} ; 175,4 \pm 5,6 \mathrm{~cm})$, praticantes de programas supervisionados de musculação para hipertrofia muscular, com experiência mínima de um ano nos exercícios analisados. Antes da coleta de dados, os voluntários responderam ao questionário PAR-Q e assinaram um termo de participação consentida, conforme Resolução 196/96 do Conselho Nacional de Saúde. Foram excluídos do experimento indivíduos usuários de medicamentos e de recursos ergogênicos e também aqueles que apresentassem problemas osteomioarticulares que pudessem influenciar a realização dos exercícios propostos. $\mathrm{O}$ projeto de pesquisa foi aprovado pelo Comitê de Ética da UCB-RJ com o protocolo de número 0074/2008.

\section{Procedimentos}

Os indivíduos foram submetidos a quatro sessões de coleta de dados, sendo duas delas destinadas à determinação da carga para 8RM dos dois exercícios propostos, e duas para aplicação dos protocolos experimentais. Foram coletados os números de RM e PSE, no final de cada série, nos exercícios supino reto com barra livre (SR) e agachamento no smith (AG). Nos primeiros dois dias, foram realizados o teste e o reteste de $8 \mathrm{RM}$ para o SR e o AG com intervalo de 48 horas entre as visitas. Os indivíduos foram divididos aleatoriamente e realizaram um sorteio para ordem de entrada nas duas situações experimentais, o que possibilitou que sete voluntários começassem pelo intervalo passivo e sete pelo intervalo com alongamento estático. A escolha da ordem de execução dos exercícios nos 
testes foi feita de forma aleatória e alternada. Após a determinação da carga para 8RM, foi respeitado um intervalo de 48 horas para aplicação das duas sessões de protocolos experimentais. As sessões envolviam três séries no SR e no AG, com intervalos entre as séries de dois minutos, realizados de forma passiva ou incluindo 30 segundos de exercícios específicos de alongamento para os grupos musculares envolvidos nos exercícios. As duas sessões foram realizadas com intervalo de 48 horas e a escolha do tipo de intervalo utilizado em cada sessão foi feita de forma alternada para os participantes.

\section{Teste e reteste de 8RM}

Tendo em vista que na literatura não existe um protocolo especifico para a realização do teste de 8RM, realizamos uma adaptação na metodologia do teste de uma repetição máxima ${ }^{17}$. O protocolo do teste consistiu em: a) um aquecimento com oito repetições com $60 \%$ da carga máxima percebida para $8 \mathrm{RM}$; b) após um minuto de repouso, os indivíduos realizaram cinco repetições com $80 \%$ do máximo percebido para $8 \mathrm{RM}$; c) após um minuto de repouso, deu-se início ao teste de carga, no qual cada indivíduo realizou no máximo cinco tentativas para cada exercício, com intervalos de cinco minutos entre as tentativas; d) quando o avaliado não conseguia mais realizar o movimento de forma correta o teste era interrompido, sendo registrada como carga máxima para oito repetições, aquela obtida na última execução completa em falha muscular concêntrica.

Após obtenção da carga do primeiro exercício, um intervalo de 10 minutos foi adotado antes de passar ao exercício seguinte. Após 48 horas do primeiro dia, foi aplicado o reteste para a verificação da reprodutibilidade da carga máxima (8RM). A escolha da ordem de execução dos exercícios nos testes foi feita de forma alternada, sendo mantida a mesma ordem durante todo procedimento experimental.

Visando reduzir a margem de erro no teste de 8RM, foram adotadas as seguintes estratégias: a) instruções padronizadas e familiarização antes do teste, de modo que todo avaliado estivesse ciente da rotina que envolve a coleta de dados; b) o avaliado foi instruído sobre técnicas de execução do exercício; c) o avaliador estava atento quanto à posição adotada pelo praticante no momento da medida, pois pequenas variações do posicionamento das articulações envolvidas no movimento podem acionar outros músculos, levando a interpretações errôneas dos escores obtidos; d) estímulos verbais foram utilizados a fim de manter alto o nível de estimulação; e) os pesos adicionais utilizados foram previamente aferidos em balança de precisão.

Foi considerada como a carga de 8RM a maior carga estabelecida em ambos os dias de testes com diferença menor que 5\%. No caso da diferença maior, os sujeitos compareciam ao local mais uma vez para a realização de novo teste, para que o cálculo da diferença fosse refeito. A carga foi considerada válida para $8 \mathrm{RM}$ quando o indivíduo, utilizando a própria força, sem colaboração externa, conseguia realizar a última repetição de forma completa. Para facilitar a realização dos exercícios, foram estabelecidas a posição inicial, fase concêntrica e fase excêntrica como etapas de execução. A descrição detalhada da posição inicial e fase concêntrica de cada exercício são apresentadas a seguir, sendo a fase excêntrica dos dois exercícios realizada a partir do final da fase concêntrica até a posição inicial.

AG: A) Posição inicial - na fase excêntrica do movimento sua execução era iniciada com os joelhos em extensão completa; b) Posição intermediária - na fase concêntrica do movimento, com os joelhos formando um ângulo de $90^{\circ}$ de flexão, com o fêmur em paralelo ao solo (limitador de amplitude), retornando então a posição inicial.

SR: a) Posição inicial - na fase excêntrica do movimento sua execução era iniciada com os cotovelos em extensão completa; b) Posição intermediária - fase concêntrica do movimento, com os cotovelos formando um ângulo de $90^{\circ}$ de flexão, com o úmero em paralelo ao solo (limitador de amplitude), retornando, então, a posição inicial.

\section{Procedimento de coleta de dados}

Os protocolos experimentais consistiram em duas sessões de exercícios para cada voluntário. Ao início de cada sessão foi feito um aquecimento com 30\% da carga de 8RM em 20 repetições. Após três minutos, foi ajustada a carga para 8RM, e os voluntários realizaram três séries no SR e no AG, sendo todas as séries executadas até o alcance da falha muscular concêntrica, mantendo o padrão de execução. Foram adotados intervalos entre as séries de dois minutos realizados de forma passiva (GIP) ou incluindo 30 segundos de exercícios específicos de alongamento estático (GAL) para os grupos musculares envolvidos nos exercícios. As duas sessões foram realizadas com intervalo de 48 horas, e a escolha do tipo de intervalo utilizado em cada sessão foi feita de forma aleatória para os participantes. $\mathrm{O}$ intervalo entre o exercício $\mathrm{SR}$ e $\mathrm{AG}$ nas duas sessões foi de cinco minutos. Os exercícios de alongamento estático utilizados no experimento 
envolveram os músculos que compõem a cintura escapular e os extensores do joelho. Abaixo, segue descrição detalhada dos movimentos utilizados para a realização dos exercícios:

Cintura escapular - com o voluntário em pé, era realizada uma abdução horizontal da articulação glenoumeral até a posição de ligeiro desconforto. O movimento foi realizado com os voluntários mantendo as mãos com polegares para cima e cotovelos totalmente estendidos.

Extensores do joelho - com o voluntário em posição pronada e com o quadril estabilizado, era realizada a flexão do joelho em conjunto com a extensão do quadril, sendo o movimento realizado até a posição de ligeiro desconforto.

Esses procedimentos foram realizados entre a primeira e segunda séries e entre a segunda e terceira séries. O número de RM e a PSE foram coletados ao final de cada série. Para a verificação da PSE, foi utilizada a escala OMNI-RES para exercício contra resistência em adultos ${ }^{13}$. Foi feita uma familiarização da escala durante as sessões dos testes de carga de 8RM. Visando reduzir a margem de erro durante todo o procedimento experimental, todos os voluntários compareciam para a realização dos testes na mesma hora do dia, na parte da manhã.

\section{Tratamento estatístico}

Para testar a reprodutibilidade das cargas entre o teste e o reteste de 8RM, foi realizado o coeficiente de correlação intraclasse (supino, $r=0,96$; agachamento, $r=0,94$ ).

O teste de Kolmogorov-Smirnov foi utilizado para testar a gaussianidade das variáveis dependentes de caracterização da amostra e das variáveis independentes. Para analisar o efeito dos diferentes tipos de intervalos no desempenho do número de repetições, foi realizada uma análise de variância (ANOVA). Para determinar as diferenças específcas, foi realizado o teste post hoc de Bonferoni. Os dados não paramétricos da PSE foram analisados através do teste de Wilcoxon. O teste $t$ pareado foi utilizado para comparar as médias do volume total do número de repetições ao final das três séries. As análises estatísticas foram realizadas a partir do pacote de programas estatísticos SPSS 14.0 (SPSS Inc., EUA). Para todas as análises, foi adotado um nível crítico de significância de $\mathrm{p}<0,05$. $O$ limite de concordância entre as sessões de teste e reteste em que ocorreu a suposta estabilização da força muscular, em cada um dos dois exercícios investigados, foi analisado mediante os procedimentos propostos por Bland \& Altman ${ }^{18}$.

\section{RESULTADOS}

As figuras 1 e 2 apresentam as plotagens propostas por Bland \& Altman ${ }^{18}$ para verificação dos limites de concordância entre as medidas obtidas nas sessões, nas quais se configurou estatisticamente o processo de estabilização das cargas. Em algumas situações, a análise de correlação intraclasse isoladamente, não se torna o procedimento mais adequado, pois podem existir altas correlações mesmo com diferenças significativas sendo apresentadas. Nesse sentido, a plotagem de Bland \& Altman ${ }^{18}$ se torna o tratamento mais robusto e confiável a ser adotado. As figuras 1 e 2 demonstram as diferenças individuais (eixo $\mathrm{Y}$ ) nos testes de 8RM, entre as sessões um e dois, em função das médias entre as duas sessões (eixo X) para os exercícios SR e AG, respectivamente.

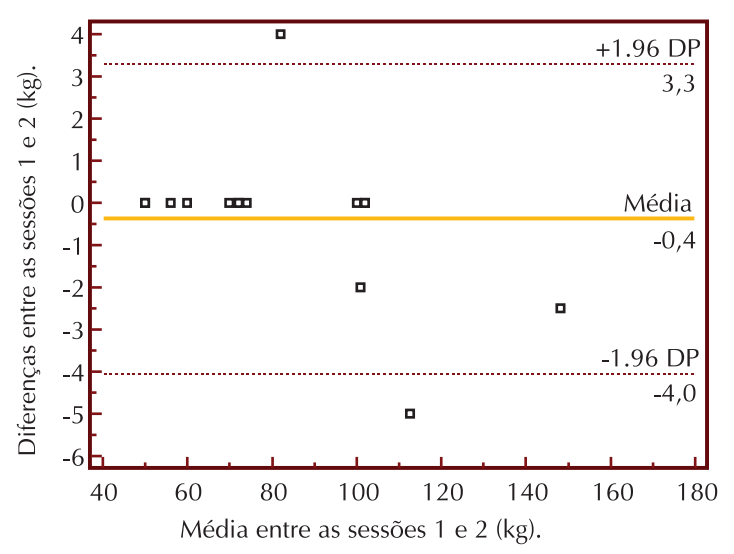

Figura 1. Plotagem de Bland \& Altman para comparações entre as sessões um e dois dos testes de 8RM para o exercício SR $(\mathrm{n}=14)$.

Nota - dois pontos estão sobrepostos.

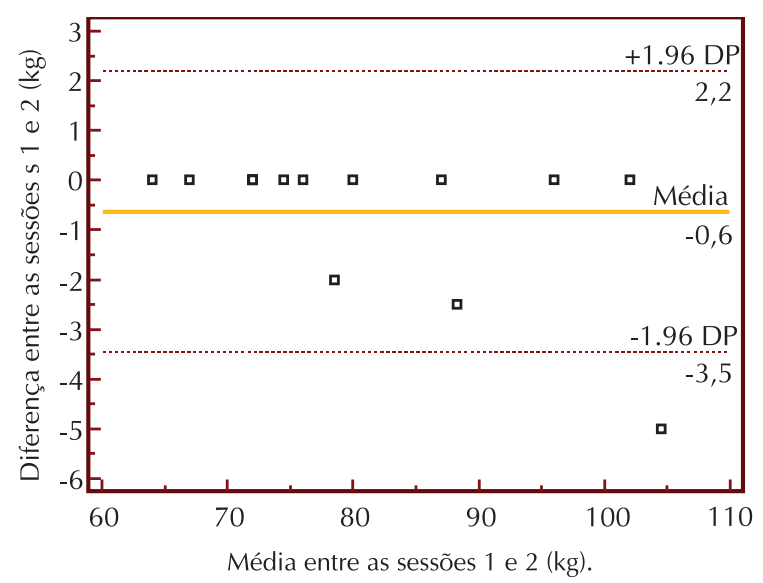

Figura 2. Plotagem de Bland \& Altman para comparações entre as sessões um e dois dos testes de 8RM para o exercício AG $(n=14)$.

Nota - dois pontos estão sobrepostos.

No exercício SR, o resultado da ANOVA na análise intergrupos (GIP vs GAL), ocorreram dife- 
renças estatisticamente significativas $(\mathrm{p}<0,05)$ na segunda (GIP $=6 \pm 0,8$ repetições vs $\mathrm{GAL}=5,2 \pm$ 1,0 repetições) e na terceira (GIP $=4,1 \pm 0,8$ repetições vs $\mathrm{GAL}=3,3 \pm 0,6$ repetições) séries (Figura 3). $\mathrm{Na}$ análise intragrupos (GIP vs GIP e GAL vs GAL), observa-se que ocorreram diferenças significativas $(\mathrm{p}<0,05)$ entre todas as séries (Figura 3).

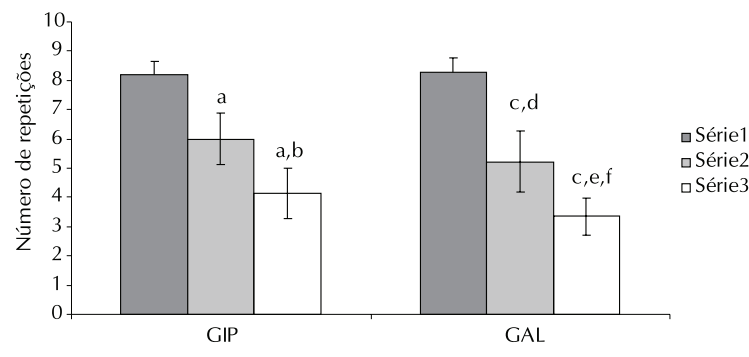

Figura 3. média e desvio padrão para o número de repetições nas condições experimentais GIP e GAL, no exercício SR ( $\mathrm{n}=$ 14). ${ }^{a}$ Diferença significativa em relação à série 1 (GIP vs GIP) $(p<0,05)$. b'Diferença significativa em relação à série 2 (GIP vs GIP) $(p<0,05)$. 'Diferença significativa em relação à série 1 (GAL vs GAL) $(\mathrm{p}<0,05)$. ${ }^{\mathrm{d}}$ Diferença significativa em relação à série 2 (GIP vs GAL) ( $p<0,05)$. eDiferença significativa em re-

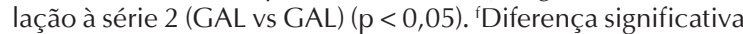
em relação à série 3 (GIP vs GAL) $(p<0,05)$. GIP = grupo que realizou intervalo passivo. $\mathrm{GAL}=$ grupo que realizou intervalo com alongamento.

No exercício AG, o resultado da ANOVA na análise intergrupos (GIP vs GAL), ocorreram diferenças estatisticamente significativas $(p<0,05)$ apenas na terceira série (GIP $=4,9 \pm 0,8$ repetições vs $\mathrm{GAL}=4,2 \pm 1,0$ repetições), conforme pode ser observado na Figura 4. Na análise intragrupos (GIP vs GIP e GAL vs GAL), observa-se que ocorreram diferenças significativas $(\mathrm{p}<0,05)$ entre todas as séries (Figura 4).

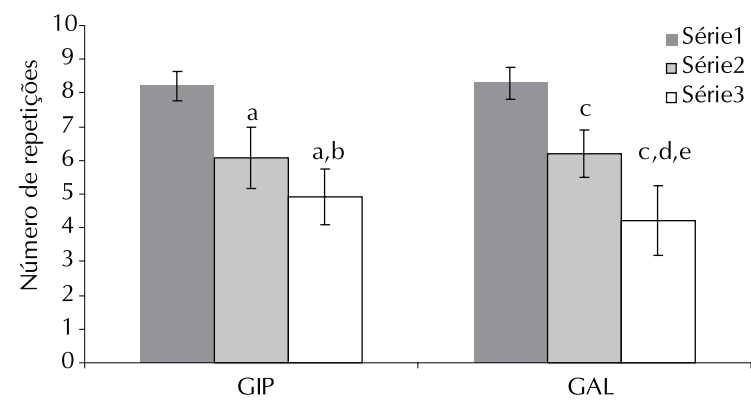

Figura 4. média e desvio padrão para o número de repetições nas condições experimentais GIP e GAL, no exercício AG $(n=$ 14). ${ }^{a}$ Diferença significativa em relação à série 1 (GIP vs GIP) $(p<0,05)$. ${ }^{b}$ Diferença significativa em relação à série 2 (GIP vs GIP) $(p<0,05)$. 'Diferença significativa em relação à série 1 (GAL vs GAL) $(p<0,05)$. 'Diferença significativa em relação à série $2(G A L$ vs GAL) $(p<0,05)$. e Diferença significativa em relação à série 3 (GIP vs GAL) $(p<0,05)$. GIP = grupo que realizou intervalo passivo. $\mathrm{GAL}=$ grupo que realizou intervalo com alongamento.

$\mathrm{Na}$ análise da PSE, os resultados do teste de Wilcoxon apresentaram diferenças estatisticamen- te significativas $(\mathrm{p}<0,05)$ tanto para o GIP quanto para o GAL entre todas as séries nos exercícios SR e AG (Tabela 1).

\begin{tabular}{lccccc}
\hline & $\begin{array}{c}\text { GIP } \\
\text { Mediana } \\
\text { Amplitude }\end{array}$ & $\begin{array}{c}\text { GAL } \\
\text { Mediana } \\
\end{array}$ & $\begin{array}{c}\text { Amplitude } \\
\text { Interquartis }\end{array}$ & $\begin{array}{c}\text { Valor- } \\
\text { Interquartis }\end{array}$ \\
\hline Supino Reto (kg) & 6 & 0,75 & 6,5 & 1,75 & $<0,05$ \\
Série 1 & $8^{\mathrm{a}}$ & 1,00 & $9^{\mathrm{a}}$ & 1,00 & $<0,05$ \\
Série 2 & $10^{\mathrm{a}, \mathrm{b}}$ & 0,00 & $10^{\mathrm{a}, \mathrm{b}}$ & 0,00 & $<0,05$ \\
Série 3 & & & & & \\
Agachamento (kg) & & & & & \\
Série 1 & 7 & 0,00 & 7 & 0,75 & $<0,05$ \\
Série 2 & $9^{\mathrm{a}}$ & 0,00 & $9^{\mathrm{a}}$ & 1,00 & $<0,05$ \\
Série 3 & $10^{\mathrm{a}, \mathrm{b}}$ & 0,00 & $10^{\mathrm{a}, \mathrm{b}}$ & 0,00 & $<0,05$ \\
\hline
\end{tabular}

${ }^{\mathrm{a}} \mathrm{p}<0,05$ vs série $1 .{ }^{\mathrm{b}} \mathrm{p}<0,05$ vs série 2 . GIP = grupo que realizou intervalo passivo. $\mathrm{GAL}=$ grupo que realizou intervalo com alongamento.

Na análise do volume total do número de repetições (Figura 5), os resultados do teste $t$ pareado apresentaram diferenças significativas $(\mathrm{p}<$ 0,05) apenas para o exercício SR (GIP $=18,3 \pm 1,5$ repetições vs $\mathrm{GAL}=16,8 \pm 1,6$ repetições). Para o exercício AG não foram observadas diferenças significativas $(\mathrm{p}>0,05)$ quando comparados os grupos $(\mathrm{GIP}=19,2 \pm 1,8$ repetições vs $\mathrm{GAL}=18,7$ $\pm 1,5$ repetições).

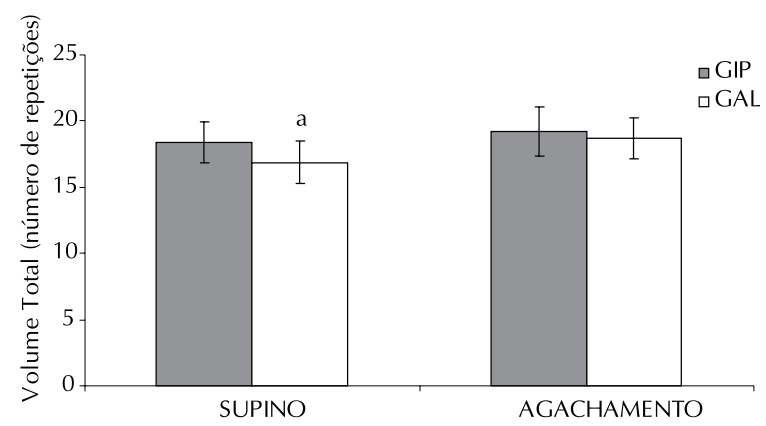

Figura 5. média e desvio padrão para o número total de repetições nas condições experimentais GIP e GAL $(n=14)$. ${ }^{a}$ Diferença significativa em relação ao grupo GIP $(p<0,05)$. $\mathrm{GIP}=$ grupo que realizou intervalo passivo. $\mathrm{GAL}=$ grupo que realizou intervalo com alongamento.

\section{DISCUSSÃO}

Os resultados do presente estudo demonstraram haver diminuição do desempenho do número de repetições quando comparados os intervalos, sendo realizados de forma passiva e com exercícios de alongamento. Neste sentido, para o exercício SR, foram observadas diferenças significativas de 18,9\% e 13,3\% nas séries dois e três respectivamente, quando comparados os 
GIP e GAL. Para o exercício AG, ocorreram diminuições significativas de $14,4 \%$ somente na terceira série, quando comparados GIP e GAL.

Através dos resultados obtidos, pôde-se observar que para o exercício SR o desempenho encontrou-se diminuído na segunda e terceira séries, enquanto no AG houve diferença significativa apenas na terceira série. Para o AG, este fenômeno pode ser explicado por dois motivos: a) devido à mecânica de movimento englobar uma quantidade muito maior de músculos agonistas e sinergistas (acessórios) que podem não ter sofrido, num primeiro momento, interferência do exercício de alongamento. Apenas com a somação de estímulos de alongamento, estes sofreram diminuição do desempenho; b) pela utilização do quadríceps, que é um grande grupamento muscular. Cramer et al. ${ }^{19}$, analisando as diferentes fases de execução do exercício de extensão do joelho, observaram haver diminuição do desempenho apenas na fase concêntrica do movimento. Porém, os autores ${ }^{19}$ utilizaram um volume de estímulos de alongamento maior do que o do presente estudo e escolheram um exercício monoarticular (menos musculatura envolvida). $O$ fato de termos utilizado um grupo muscular maior em um exercício multiarticular pode ter ocasionado uma influência negativa do desempenho concêntrico, apenas com a soma dos estímulos de alongamento, interferindo, assim, somente na terceira série do exercício. Os dados do presente estudo corroboram os achados de Cramer et al. ${ }^{19}$ Os autores utilizaram quatro exercícios de alongamento com quatro séries e trinta segundos de sustentação na posição de alongamento. No presente estudo, mesmo utilizando um volume menor de exercícios de alongamento, pôde ser observada também uma diminuição da força.

Diversos estudos ${ }^{5-7,19,20}$ demonstraram que exercícios de alongamento interferem negativamente, de forma aguda, no desempenho da força muscular quando realizados imediatamente antes da atividade principal. A redução da tensão passiva e da rigidez da unidade miotendinosa que ocorre após exercícios de alongamento pode dificultar a transferência de força do tendão para o músculo ${ }^{21}$, ocasionando assim uma diminuição do desempenho. Após a realização de repetitivos e prolongados alongamentos passivos, Avela et al. ${ }^{20}$ observaram um decréscimo na atividade elétrica e na contração voluntária máxima entre 7,6\% e 16,8\% nos músculos gastrocnêmios e solear. Os autores observaram que ocorreram modificações no sistema aponeurosetendão, assim como um fenômeno conhecido como relaxamento de estresse e/ou deformações plásticas, que afetam a resposta proprioceptiva, diminuindo a ativação das unidades motoras.

Gomes et al. ${ }^{22}$ verificaram diminuições no desempenho do teste de 1RM para os métodos estático e FNP no exercício supino. A utilização de cargas máximas e o alto volume do número de séries utilizadas pelos autores podem diminuir a aplicabilidade dos resultados. Porém, mesmo com um menor volume de séries de exercícios de alongamento e menor intensidade de carga utilizada, os dados do presente estudo corroboram os dados de Gomes et al. ${ }^{22}$, demonstrando que para o desempenho de altas intensidades (1RM e 8RM) há uma influência negativa dos exercícios de alongamento quando realizados antes do exercício supino.

A variação do número de séries, bem como o tempo de manutenção na posição de alongamento são bastante diferentes na literatura e, por muitas vezes, extrapolam em muito o recomendado ${ }^{23}$. Fowles et al. ${ }^{5} \mathrm{e}$ Behm et al..$^{24}$ utilizaram 135 e 45 segundos de manutenção na posição de alongamento e observaram diminuições de $25 \%$ e $12 \%$, respectivamente, no desempenho das contrações voluntárias máximas. No presente estudo, apesar de ter sido utilizado um tempo inferior de manutenção na posição de alongamento (30 segundos), pôde-se observar também diminuições no desempenho do número de repetições entre 13,3\% e 18,9\%. Esta diminuição pode ocorrer devido a um fenômeno conhecido como fadiga neural, em que ocorre uma diminuição da ativação das unidades motoras em concomitância com a diminuição da atividade elétrica do músculo5.

Duas hipóteses são desenvolvidas para explicar porque a flexibilidade diminui de forma aguda o desempenho da força muscular: fatores mecânicos, que provocam alterações nas propriedades viscoelásticas da unidade miotendinosa; e fatores neurais, com a diminuição da ativação das unidades motoras. Avela et al..$^{25}$ observaram reduções na sensitividade dos fusos musculares, que acabam por diminuir a atividade das fibras aferentes de grande calibre, juntamente com uma inibição dos motoneurônios alfa gerada pelos receptores articulares tipos III e IV ${ }^{26}$, que reduziram em 23,2\% a CVM da musculatura do tríceps sural. Alterações nas propriedades viscoelásticas da unidade miotendinosa reduzem a tensão passiva e a rigide $z^{21,27}$. Como uma das funções do tendão é transferir a força produzida pela musculatura esquelética para os ossos e articulações, uma unidade miotendinosa menos rígida transmitirá de forma menos eficiente as alterações 
de tensão na musculatura. Tais alterações viscoelásticas podem colocar o componente contrátil em uma posição menos favorável em termos de produção de força, nas curvas de força-comprimento e força-velocidade, que acarreta, consequentemente, em uma insuficiente transmissão de força do músculo para o sistema esquelético ${ }^{21,27}$.

No que diz respeito à utilização da PSE para o acompanhamento da intensidade do esforço em experimentos que observam a influência de distintos intervalos entre as séries, apenas um experimento foi encontrado ${ }^{12}$. Woods et al. ${ }^{12}$ examinaram os efeitos de diferentes tempos de intervalo sobre a PSE, durante três séries, na cadeira extensora. Foram voluntários deste experimento, 30 indivíduos saudáveis (15 homens; 15 mulheres), que executaram três séries de 10 repetições, a 70\% de 10RM, com um, dois ou três minutos de intervalo entre as séries. A PSE foi registrada através da escala de Borg (CR10) depois de cada série. Os resultados não demonstraram nenhuma diferença significativa da PSE em relação ao tempo de intervalo. Entretanto, revelaram um valor de PSE, significativamente, mais alto da terceira série, em relação à segunda e à primeira série em todos os intervalos. No presente estudo, apesar de a PSE ter sido observada apenas ao final de cada série e ter sido utilizada a escala OMNI-RES de PSE ${ }^{13-14}$, os resultados encontrados foram similares aos obtidos por Woods et al..$^{12} \mathrm{~A}$ PSE não apresentou diferenças em relação aos diferentes tipos de intervalo utilizados, porém apresentou aumentos da segunda em relação à primeira série e da terceira em relação à primeira e segunda séries para ambos os intervalos. Os resultados da PSE demonstram que ambos os tipos de intervalo repercutem de forma similar sobre o acúmulo de fadiga, e que essa fadiga apresenta aumentos durante a progressão das séries.

Em relação a diferentes tipos de intervalo, Caruso e Coday ${ }^{16}$ observaram que o intervalo de um minuto, realizado de forma passiva, foi mais eficiente para o aumento do desempenho do volume total do número de repetições, quando comparado com intervalo passivo de trinta segundos e com intervalo passivo realizando massagem e elevação corporal. Os autores examinaram os efeitos dos diferentes tipos de intervalo no desempenho do volume total do número de repetições nos exercícios leg press, mesa flexora, desenvolvimento e rosca bíceps. Foram voluntários deste experimento 30 sujeitos (27 homens; três mulheres), que realizaram oito séries em todos os exercícios, com as cargas variando entre $50 \%$ e $90 \%$ de 1RM. Em nosso estudo, o grupo experimental realizou o intervalo de forma passiva (dois minutos) para o exercício supino e agachamento. Para o supino, foi observado um maior desempenho do volume total do número de repetições, quando comparado com intervalo realizando o alongamento estático, corroborando os dados de Caruso e Coday ${ }^{16}$. Mesmo para o exercício agachamento, apesar de não haver diferença significativa, pôde ser observada uma leve tendência de um melhor desempenho no GIP. Talvez, com um volume maior de séries, essa diferença pudesse ser maximizada, como foi realizado pelos autores ${ }^{16}$.

Após realizar uma revisão da literatura, a principal hipótese levantada pelos autores era que ocorreria uma diminuição no desempenho da força ao ser precedida por exercício de alongamento. Os resultados do presente estudo confirmam a hipótese e somam-se aos dados já existentes na literatura, mostrando haver uma diminuição no desempenho da força, independentemente do segmento corporal envolvido. E ainda, mostram que apenas um único exercício de alongamento foi suficiente para interferir no desempenho da força muscular. Os dados do presente estudo não corroboram os dados de Beedle et al. ${ }^{28}$, que apesar de utilizarem um maior volume de exercícios de alongamento estático, não encontraram diferenças significativas no desempenho do teste de 1RM para os exercícios supino e leg press. Entretanto, os autores ${ }^{28}$ utilizaram em seu experimento um grupo de voluntários com diferentes níveis de treinamento, isto pode ter comprometido a validade externa dos dados. Vale frisar que, apesar de não observarem uma diminuição estatisticamente significativa no desempenho da força em 1RM, verifica-se nos resultados que ocorreu uma pequena diminuição dos níveis da força.

Apesar de terem sido tomados cuidados acerca de todo o procedimento de coleta com o objetivo de aumentar a validade do estudo, uma das limitações foi a não realização da familiarização dos indivíduos com o teste de 8RM, possibilitando, assim, haver influência no desempenho dos voluntários, pelo processo de aprendizagem com o teste. Na literatura, não foram encontrados estudos que testem a reprodutibilidade em testes de 8RM. Os artigos científicos investigam apenas o teste de 1RM, concluindo que a estabilização da carga para 1RM varia de acordo com o sexo, tipo exercício e faixa etária analisada. Dias et al. ${ }^{29}$, utilizando os mesmos exercícios do presente estudo com homens jovens, concluíram que de 2 a 3 sessões, são suficientes em testes de 1RM para indivíduos com apenas 6 meses de experiência em treinamento de força. Levinger 
et $a .^{30}$ utilizando em sua amostra homens e mulheres adultos, concluíram que uma única sessão foi suficiente para estabilização da carga de 1RM para o exercício supino $\left(\mathrm{r}^{2}=0,99\right)$ e para o exercício leg press, duas sessões foram suficientes $\left(\mathrm{r}^{2}=0,99\right)$. No presente estudo, foi realizado o teste de 8RM que se torna mais fácil de aprender devido a sua equivalência com a situação real de treinamento dos nossos voluntários, pois todos possuíam, pelo menos, um ano de experiência com o treinamento, o que representa o dobro de experiência em treinamento de força do estudo acima citado.

A outra limitação foi em função da posição de alongamento adotada. É importante enfatizar que a posição de alongamento utilizada para a musculatura do peitoral, apesar de ser bastante comum nas academias de ginástica e em clubes, provavelmente, interferiu na resposta da produção de força. Com os cotovelos estendidos não se isola o alongamento para a musculatura do peitoral. No entanto, mesmo com essa limitação do estudo, foi observada uma diminuição no desempenho da força. A hipótese é que, com o exercício de alongamento sendo conduzido com os cotovelos flexionados (isolando assim o grupamento muscular alongado), a diminuição no desempenho da força poderá ser ainda maior, pois os músculos acessórios não sofreriam o impacto do exercício de alongamento e o músculo peitoral seria alongado em uma amplitude maior, maximizando o efeito do alongamento. Faz-se necessária a elaboração de outros estudos que envolvam diferentes variáveis, entre elas: o número de séries, o tempo de sustentação da posição de alongamento e populações com diferentes idades. Sugere-se, também, que se observem os efeitos crônicos sobre as variáveis apresentadas.

A repercussão destes resultados pode auxiliar os profissionais da área da saúde, tanto aquele que prescreve treinamento para as atividades físico-esportivas quanto aquele que trabalha com a reabilitação, no sentido de alertar sobre os efeitos dos exercícios de alongamento realizados antes das séries de exercícios de força. Ainda mais, quando as atividades físico-desportivas dependerem diretamente do desempenho da força.

\section{CONCLUSÃO}

De forma aguda, assumindo-se as limitações deste estudo e as características do grupo amostral, podese inferir que quando comparados os intervalos passivos com os intervalos utilizando exercício de alongamento, observou-se que pode haver diminuição do número de repetições máximas no decorrer de três séries, quando se utiliza o exercício de alongamento entre as séries. Para o exercício SR, ocorreram diminuições na segunda e terceira séries, enquanto que para o AG essa diminuição somente foi observada na terceira série. $\mathrm{O}$ volume total do número de repetições apresentou diferença apenas para o SR, no qual o GAL totalizou um número menor de repetições quando comparado com GIP. A PSE aumentou em conformidade com o acúmulo do número de séries independentemente do intervalo utilizado. Conclui-se que o exercício de alongamento estático, quando realizado entre as séries, pode provocar diminuição no desempenho da força em séries múltiplas com carga ajustada para 8RM.

\section{REFERÊNCIAS BIBLIOGRÁFICAS}

1. Hill-Haas S, Bishop D, Dawson B, Goodman C, Edge J. Effects of rest interval during high-repetition resistance training on strength, aerobic fitness, and repeatedsprint ability. J Sports Sci 2007;25(6):619-628.

2. Ahtiainen JP, Pakarinen A, Alen M, Kraemer WJ, Häkkinen K. Short vs. Long rest period between the sets in hypertrophic resistance training: influence on muscle strength, size, and hormonal adaptations in trained men. J Strength Cond Res 2005;19(3):572-582.

3. Willardson JM, Burkett IN. A comparison of 3 different rest intervals on the exercise volume completed during a workout. J Strength Cond Res 2005;19(1):23-26.

4. Willardson JM, Burkett IN. The effect of rest interval length on bench press performance with heavy vs. Light load. J Strength Cond Res 2006;20(2):396-409.

5. Fowles JR, Sale DG, MacDougall JD. Reduced strength after passive stretch of the human plantarflexors. J Appl Physiol 2000;89(3):1179-1188.

6. Power K, Behm D, Cahill F, Carroll M, Young W. An acute bout of static stretching: effects on force and jump performance. Med Sci Sports Exerc 2004;36(8):1389-1396.

7. Marek SM, Cramer JT, Fincher AL, Massey LL, Dangelmaier SM, Purkayastha S, et al. Acute effects of static and proprioceptive neuromuscular facilitation stretching on muscle strength and power output. J Athletic Training 2005;40(2):94-103.

8. Ogura Y, Miyahara Y, Naito H, Katamoto S, Aoki I. Duration of static stretching influences muscle force production in hamstring muscles. J Strength Cond Res 2007;21(3):788-792.

9. Egan AD, Cramer JT, Massey LL, Marek SM. Acute effects of static stretching on peak torque and mean power output in national collegiate athletic association division I women's basketball players. J Strength Cond Res 2006;20(4):778-782.

10. Unick J, Kieffer S, Cheesman W, Feeney A. The acute effects of static and ballistic stretching on vertical jump performance in trained women. J Strength Cond Res 2005;19(1):206-212. 
11. Yamaguchi T, Ishii K. Effects of static stretching for 30 seconds and dynamic stretching on leg extension power. J Strength Cond Res 2005;19(3):677-683.

12. Woods S, Bridge T, Nelson D, Risse K, Pincivero DM. The effects of rest interval length on ratings of perceived exertion during dynamic knee extension exercise. J Strength Cond Res 2004;18(3):540-545.

13. Lagally KM, Robertson RJ. Construct validity of the omni resistance exercise scale. J Strength Cond Res 2006;20(2):252-256.

14. Robertson RJ, Goss FL, Rutkowski J, Lenz B, Dixon $\mathrm{C}$, Timmer J, et al. Concurrent validation of the omni perceived exertion scale for resistance exercise. Med Sci Sports Exerc 2003;35(2):333-341.

15. Simão R, Farinati PTV, Polito MD, Viveiros L, Fle$\mathrm{ck}$ SJ. Influence of exercise order on the number of repetitions performed and perceived exertion during resistance exercise in women. J Strength Cond Res 2007;21(1):23-28.

16. Caruso JF, Coday MS. The combined acute effects of massage, rest periods and body part elevation on resistance exercise performance. J Strength Cond Res 2008;22(2):575-582.

17. American College of Sports Medicine. Diretrizes do ACSM Para os Testes de Esforço e sua Prescrição. 6a ed. Rio de Janeiro: Guanabara Koogan, 2003.

18. Bland JM, Altman DJ. Regression Analysis. Lancet 1986;1(8486):908-909.

19. Cramer JT, Housh TJ, Coburn JW, Beck TW, Johnson GO. Acute effects of static stretching on maximal eccentric torque production in women. J Strength Cond Res 2006;20(2):354-358.

20. Avela J, Finni T, Liikavainio T, Niamelä E, Komi PV. Neural and mechanical responses of the triceps surae muscle group after $1 \mathrm{~h}$ of repeated passive stretches. J Appl Physiol 2004;96(6):2325-2332.

21. Wilson GL, Murphy AI, Pryor JF. Muscle tendinous stiffness: its relationship to eccentric, isometric and concentric performance. J Appl Physiol 1994;76(6):2714-2719.

22. Gomes TM, Rubini EC, Junior HSN, Novaes JS, Trindade A. Efeito agudo dos alongamentos estático e fnp sobre o desempenho da força dinâmica máxima. Rev Bras Fisiol Exerc 2005;4(1):13-16.

23. American College of Sports Medicine. The recommended quantity and quality of exercise for developing and maintaining cardiorespiratory and muscular fitness, and flexibility in healthy adults. Position stand. Med Sci Sports Exerc 1998;30(6):975-991.

24. Behm DG, Button DC, Butt JC. Factors affecting force loss with prolonged stretching. Can J Appl Physiol 2001;26(3):262-272.

25. Avela J, Kyröläinen H, Komi PV. Altered reflex sensitivity after repeated and prolonged passive muscle stretching. J Appl Physiol 1999;86(4):1283-1291.

26. Avela J, Kyröläinen H, Komi PV, Rama D. Reduced reflex sensitivity persists several days after long-lasting stretch-shortening cycle exercise. J Appl Physiol 1999;86(4):1292-1300.

27. Kubo K, Kanehisa H, Kawakami Y, Fukunaga T. Influence of static stretching on viscoelastic properties of human tendon structures in vivo. J Appl Physiol 2001;90(2):520-527.

28. Beedle B, Rytter SJ, Healy RC, Ward TR. Pretesting static and dynamic stretching does not affect maximal strength. J Strength Cond Res 2008;22(6):1838-1843.

29. Dias RMR, Cyrino ES, Salvador EP, Caldeira LFS, Nakamura FY, Papst RR, et al. Influência do processo de familiarização para avaliação da força muscular em testes de 1-RM. Rev Bras Med Esp 2005;11(1):34-38.

30. Levinger I, Goodman C, Hare DL, Jerums G, Toia D, Selig S. The reliability of the 1RM strength test for untrained middle-aged individuals. J Sci Med Sport 2009;12(2):310-316.

\section{Endereço para correspondência}

Jefferson da Silva Novaes

Avenida Prefeito Dulcídio Cardoso 11100, apto

1901 - Barra da Tijuca

CEP: 21793-012, Rio de Janeiro, RJ - Brasil

E-mail: jsnovaes@terra.com.br 